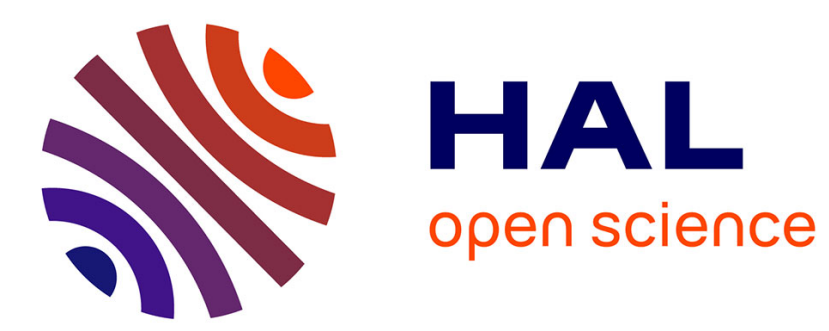

\title{
A Super Fast Attitude Determination Algorithm for Consumer-Level Accelerometer and Magnetometer
} Jin Wu, Zebo Zhou, Hassen Fourati, Yuhua Cheng

\section{To cite this version:}

Jin Wu, Zebo Zhou, Hassen Fourati, Yuhua Cheng. A Super Fast Attitude Determination Algorithm for Consumer-Level Accelerometer and Magnetometer. IEEE Transactions on Consumer Electronics, 2018, 64 (3), pp.375 - 381. 10.1109/tce.2018.2859625 . hal-01922922

\section{HAL Id: hal-01922922 \\ https://hal.inria.fr/hal-01922922}

Submitted on 14 Nov 2018

HAL is a multi-disciplinary open access archive for the deposit and dissemination of scientific research documents, whether they are published or not. The documents may come from teaching and research institutions in France or abroad, or from public or private research centers.
L'archive ouverte pluridisciplinaire HAL, est destinée au dépôt et à la diffusion de documents scientifiques de niveau recherche, publiés ou non, émanant des établissements d'enseignement et de recherche français ou étrangers, des laboratoires publics ou privés. 


\title{
A Super Fast Attitude Determination Algorithm for Consumer-Level Accelerometer and Magnetometer
}

\author{
Jin $\mathrm{Wu}^{(}{ }^{\circledR}$, Member, IEEE, Zebo Zhou ${ }^{(}$, Hassen Fourati, and Yuhua Cheng ${ }^{\circledR}$, Senior Member, IEEE
}

\begin{abstract}
A super fast attitude solution is obtained for consumer electronics accelerometer-magnetometer combination. The quaternion parameterizing the orientation is analytically derived from a least-square optimization that maintains very simple form. Like previously developed approaches, this algorithm does not require predetermined magnetometer reference vector. It has been proven in the paper that the proposed algorithm is equivalent to two recent representative methods. Computational complexity analysis shows that the proposed algorithm has the least floating-number operations. Comparisons with recent and classical methods indicate the definite superiority of the proposed algorithm on execution time in real embedded applications.
\end{abstract}

Index Terms-Attitude determination, analytical solution, accelerometer, magnetometer, quaternion.

\section{INTRODUCTION}

$\mathbf{T}$ HE MICRO-ELECTRO-MECHANICAL-SYSTEMS (MEMS) accelerometer-magnetometer combination is a key component in many consumer-level products requiring motion sensing [1]. These applications have enriched the functionalities of nowadays intelligent devices like cellphones, smart wearables, gait logger, unmanned aerial vehicles and etc. [2]-[4]. The accelerometer and magnetometer along with gyroscope can together form a general inertial measurement unit (IMU) [2]. In principle, using accelerometer and magnetometer respectively, one device can compute the attitude solution by means of local gravity and magnetic-field sensing [5]. For most consumer-level equipments, such sensor combination is sufficient for evaluation of orientation measurements [6], [7].

For nowadays accelerometer and magnetometer, there are always algorithms estimating Euler angles for motion detection on the chip. This characteristic is usually not

Manuscript received March 14, 2018; revised May 26, 2018 and July 17, 2018; accepted July 19, 2018. Date of publication July 25, 2018; date of current version September 24, 2018. This work was supported in part by the National Natural Science Foundation of China under Grant 41604025, and in part by the State Key Laboratory of Geodesy and Earths Dynamics (Institute of Geodesy and Geophysics, Chinese Academy of Sciences) under Grant SKLGED2018-3-2-E. (Corresponding author: Zebo Zhou.)

J. Wu and Z. Zhou are with School of Aeronautics and Astronautics, University of Electronic Science and Technology of China, Chengdu 611731, China (e-mail: jin_wu_uestc@hotmail.com; klinsmann.zhou@gmail.com).

H. Fourati is with CNRS, GIPSA-Lab, University Grenoble Alpes, 38400 Grenoble, France (e-mail: hassen.fourati@gipsa-lab.grenoble-inp.fr).

Y. Cheng is with the School of Automation, University of Electronic Science and Technology of China, Chengdu 611731, China (e-mail: yhcheng@uestc.edu.cn).

Color versions of one or more of the figures in this paper are available online at http://ieeexplore.ieee.org.

Digital Object Identifier 10.1109/TCE.2018.2859625 recommended for long-endurance products because of their disadvantage on high power consumption. Actually, a faster sensor fusion method leads to less power consumption and higher system reliability [8]. Existing methods calculating the attitude results have been developed in the last decade. The most representative ones are factored quaternion algorithm (FQA) and Markley's two-vector method, which both employ the geometric relationship between sensing axes [9], [10]. Some other algorithms are proposed recently, e.g., algebraic quaternion algorithm (AQUA, [11]), symbolic accelerometermagnetometer fusion [12], singular-value-decomposition (SVD) filter [13] and etc. [14].

The attitude determination results are often later processed by complementary and Kalman filters [15]-[17] for more smooth estimates. This requires a later loosely coupled fusion with gyroscope [18]. Generally speaking, to achieve the goal of extremely low power consumption, one attitude estimation algorithm should have at least 1) Energy-saving sensors; 2) Time-costly measurement equations; 3) Simple and explicit solutions. For instance, replacing gyroscopes with accelerometer and magnetometer, the power consumption can be significantly reduced accordingly since the MEMS gyroscopes are usually drived with more electricity. Recently, Makni et al. [19] have developed an orientation algorithm using the adaptive Kalman filter where the gyroscope measurements are configured to be intermittent so that the power can be saved. The left two factors are also very important to reduce computation burdens. For example, the Kalman filter also consumes much more computational resources than simple ones like complementary filters. Therefore for many embedded products, the complementary filters are usually employed for saving power.

In previous research, the fusion problem of accelerometer and magnetometer is typically solved via sophisticated mathematical results. The aforementioned representatives for accelerometer and magnetometer, e.g., FAQ, AQUA and SVD do not show very good performance for long-endurance application according to complicated internal computations, e.g., matrix manipulations, trigonometric functions, square roots and etc. Following the steps of these former endeavors, in this paper we have the following main contributions.

1) A novel fast quaternion solution is derived according to the least-square optimization.

2) The proposed attitude determination algorithm is systematically investigated showing that it owns the same accuracy as previous representatives achieve. 
3) The numerical analysis is presented which indicates the super fast computation speed of the proposed algorithm. The experiments also indicate that the new method has an evident improvement of over $50 \%$ on the execution time.

This paper is organized as follows: Section II states the problem and presents the solution. Section III discusses the equivalence of various algorithms. Section IV presents the computation complexities of representative algorithms and the proposed one. Section V contains experimental validations while Section VI gives concluding remarks.

\section{Problem Statement And Solution}

The accelerometer and magnetometer have their normalized observations $\mathbf{A}^{b}=\left(a_{x}, a_{y}, a_{z}\right)^{T}, \mathbf{M}^{b}=\left(m_{x}, m_{y}, m_{z}\right)^{T}$ respectively in the body frame $b$. Along with their normalized reference vectors $\mathbf{A}^{r}=(0,0,1)^{T}, \mathbf{M}^{r}=\left(m_{N}, 0, m_{D}\right)^{T}$ in reference frame $r$ (here is the North-East-Down) such that

$$
\left\{\begin{array}{l}
a_{x}^{2}+a_{y}^{2}+a_{z}^{2}=1 \\
m_{x}^{2}+m_{y}^{2}+m_{z}^{2}=1 \\
m_{N}^{2}+m_{D}^{2}=1
\end{array}\right.
$$

they are related by the direction cosine matrix (DCM) $\mathbf{C} \in$ $S O(3)$ with the following Wahba's optimization:

$$
\underset{\substack{\mathbf{C} \mathbf{C}^{T}=\mathbf{C}^{T} \mathbf{C}=\mathbf{I}, \operatorname{det}(\mathbf{C})=+1}}{\arg \min }\left\{w\left\|\mathbf{A}^{b}-\mathbf{C A}^{r}\right\|^{2}+(1-w)\left\|\mathbf{M}^{b}-\mathbf{C M}^{r}\right\|^{2}\right\}
$$

where $w$ is the weight of accelerometer and $1-w$ belongs to magnetometer [12]. The Wahba's problem can be solved with many existing numerical methods, e.g., QUEST, FLAE, ESOQ and etc. [20]. It is also noted that the optimization is currently solved by means of numerical methods like gradientdescent algorithm (GDA, [21], [22]), Gauss-Newton algorithm (GNA, [23]), Levenberg-Marquardt algorithm (LMA, [24]). However, according to uncertainties of these algorithms on the convergence and execution time consumption, we do not address discussion and comparison of them in this paper. By a series of transformation, it is proven that Wahba's solution is equivalent to finding the eigenvector of the maximum eigenvalue associated with the Davenport's K matrix [25]:

$$
\mathbf{K}=\left[\begin{array}{cc}
\mathbf{B}+\mathbf{B}^{T}-\operatorname{tr}(\mathbf{B}) \mathbf{I} & \mathbf{z} \\
\mathbf{z}^{T} & \operatorname{tr}(\mathbf{B})
\end{array}\right]
$$

where $\operatorname{tr}(\cdots)$ is the matrix trace; I stands for the identity matrix. Other parametric values are provided as follows

$$
\begin{aligned}
\mathbf{B} & =w \mathbf{A}^{b}\left(\mathbf{A}^{r}\right)^{T}+(1-w) \mathbf{M}^{b}\left(\mathbf{M}^{r}\right)^{T} \\
\mathbf{z}^{T} & =\left(B_{23}-B_{32}, B_{31}-B_{13}, B_{12}-B_{21}\right)
\end{aligned}
$$

in which $B_{i j}$ stands for the element of $\mathbf{B}$ in $i$-th row and $j$-th column. For the specific fusion of accelerometer and magnetometer, the above mentioned methods will not obtain results within relative execution time. The reason is that these methods are general solvers. They compute the analytical forms of the characteristic polynomial of $\mathbf{K}$ with many existing results [8]. The eigenvector can be computed until the maximum eigenvalue having been found out via numerical iterations, e.g., Gauss-Newton recursion. Therefore, we are thinking how to derive the closed-form eigenvalue of this problem and solve the optimal eigenvector directly.

Inserting vector components into the above equations, the analytic form of $\mathbf{K}$ can be computed. Then the characteristic polynomial is obtained by

$$
\operatorname{det}\left(\mathbf{K}-\lambda_{\mathbf{K}} \mathbf{I}\right)=0
$$

where

$$
\lambda_{\mathbf{K}}^{4}+\tau_{1} \lambda_{\mathbf{K}}^{2}+\tau_{2} \lambda_{\mathbf{K}}+\tau_{3}=0
$$

in which the parameter set can be determined by [26]

$$
\begin{aligned}
\tau_{1} & =-2 \operatorname{tr}^{2}(\mathbf{B})+\operatorname{tr}\left[\operatorname{adj}\left(\mathbf{B}+\mathbf{B}^{T}\right)\right]-\mathbf{z}^{T} \mathbf{z} \\
\tau_{2} & =-\operatorname{tr}[\operatorname{adj}(\mathbf{K})] \\
\tau_{3} & =\operatorname{det}(\mathbf{K})
\end{aligned}
$$

where adj denotes the adjoint matrix. In advance, the coefficients can be computed to

$$
\begin{aligned}
\tau_{1}= & -4 w^{2}+4 w-2+4 \alpha m_{D} w(w-1) \\
\tau_{2}= & 0 \\
\tau_{3}= & 4 \alpha m_{D} w(w-1)\left(-2 w^{2}+2 w-1\right) \\
& +4 w^{2}(w-1)^{2}\left(\alpha^{2}-m_{N}^{2}\right) \\
& +4 w^{4}-8 w^{3}+8 w^{2}-4 w+1
\end{aligned}
$$

with which the eigenvalues of $\mathbf{K}$ is given by

$$
\begin{aligned}
& \lambda_{\mathbf{K}, 1}=\sqrt{(1-w)^{2}+w^{2}+2 w(1-w)\left(\alpha m_{D}+V\right)} \\
& \lambda_{\mathbf{K}, 2}=\sqrt{(1-w)^{2}+w^{2}+2 w(1-w)\left(\alpha m_{D}-V\right)} \\
& \lambda_{\mathbf{K}, 3}=-\sqrt{(1-w)^{2}+w^{2}+2 w(1-w)\left(\alpha m_{D}-V\right)} \\
& \lambda_{\mathbf{K}, 4}=-\sqrt{(1-w)^{2}+w^{2}+2 w(1-w)\left(\alpha m_{D}+V\right)}
\end{aligned}
$$

where

$$
\begin{aligned}
& \alpha=a_{x} m_{x}+a_{y} m_{y}+a_{z} m_{z} \\
& V=m_{N} \sqrt{1-\alpha^{2}}
\end{aligned}
$$

The derivation details are given in the Appendix A. From the above equation, we have the identity $V>0$ since the magnetic dip angle $\theta \in\left[-\frac{\pi}{2}, \frac{\pi}{2}\right]$ ensures $m_{N}=\cos \theta>0$ [9]. Sorting these eigenvalues in descending order, it is obtained that

$$
\lambda_{\mathbf{K}, 1} \geq \lambda_{\mathbf{K}, 2}>\lambda_{\mathbf{K}, 3} \geq \lambda_{\mathbf{K}, 4}
$$

Therefore the attitude quaternion should be the eigenvector associated with the eigenvalue $\lambda_{\mathbf{K}, 1}$.

In accelerometer-magnetometer fusion, in fact the magnetic dip angle is not required before sensor setup, since [25], [27]

$$
m_{D}=\alpha, m_{N}=\sqrt{1-\alpha^{2}}
$$

always holds, with the constraint of the DCM. In this way, the roll and pitch are not affected by magnetic sensing and the 
analytic form of the maximum eigenvalue can be simplified to

$$
\begin{aligned}
\lambda_{\mathbf{K}, 1} & =\sqrt{(1-w)^{2}+w^{2}+2 w(1-w)\left(\alpha m_{D}+m_{N} \sqrt{1-\alpha^{2}}\right)} \\
& =\sqrt{(1-w)^{2}+w^{2}+2 w(1-w)\left(m_{D}^{2}+m_{N}^{2}\right)} \\
& =1
\end{aligned}
$$

Inserting the above eigenvalue back into $\mathbf{K}-\mathbf{I}$, the final results of the row echelon form can be given by

$$
\mathbf{K}-\mathbf{I} \Rightarrow\left(\begin{array}{cccc}
1 & \cdots & & a \\
& 1 & \vdots & b \\
& & 1 & c \\
& & & d
\end{array}\right)
$$

where

$$
\begin{aligned}
a= & \frac{1}{P}\left\{\begin{array}{l}
m_{y}\left[m_{D}-a_{x} m_{N}+\alpha(w-1)-w m_{D}\right] \\
+a_{y}\left[m_{N} m_{x}+m_{N}^{2}(1-w)+w-w \alpha m_{D}\right]
\end{array}\right\} \\
b= & \frac{1}{P}\left\{\begin{array}{l}
m_{N} m_{z}+\alpha m_{x}(1-w)+m_{D} m_{x}(w-1) \\
+a_{z} m_{N}\left[m_{D}(w-1)-\alpha w\right] \\
+a_{x}\left[m_{N}^{2}(w-1)-w+w \alpha m_{D}\right]
\end{array}\right\} \\
c= & \frac{1}{P}\left\{m_{N}\left[-\left(1+a_{z}\right) m_{y}+a_{y}\left(m_{D}+m_{z}+w \alpha-w m_{D}\right)\right]\right\} \\
d= & \frac{1}{P}\left\{2\left(1+\alpha^{2}-2 \alpha m_{D}-m_{N}^{2}\right)(1-w) w\right\} \\
P= & -1-m_{N} m_{x}-a_{z} m_{N} m_{x}+(w-1) a_{z} m_{N}^{2} \\
& +(w-1) m_{D} m_{z}-a_{z} w+a_{x} m_{N}\left(m_{D}+m_{z}-m_{D} w\right) \\
& +\alpha\left(m_{D}+m_{z}+a_{z} m_{D} w+a_{x} m_{N} w-m_{z} w\right)
\end{aligned}
$$

As $\alpha=m_{D}$, the fundamental solution to $(\mathbf{K}-\mathbf{I}) \mathbf{q}=\mathbf{0}$ is given by

$$
\begin{aligned}
\tilde{\mathbf{q}} & =\left(\tilde{q}_{0}, \tilde{q}_{1}, \tilde{q}_{2}, \tilde{q}_{3}\right)^{T} \\
\tilde{q}_{0} & =\left(a_{z}-1\right)\left(m_{N}+m_{x}\right)+a_{x}\left(m_{D}-m_{z}\right) \\
\tilde{q}_{1} & =\left(a_{z}-1\right) m_{y}+a_{y}\left(m_{D}-m_{z}\right) \\
\tilde{q}_{2} & =a_{z} m_{D}-a_{x} m_{N}-m_{z} \\
\tilde{q}_{3} & =-a_{y}\left(m_{N}+m_{x}\right)+a_{x} m_{y}
\end{aligned}
$$

which shows that the solution is free of pre-determined weights of vector observations. Note that the solved quaternion is a vector-wise one, i.e., [28]

$$
\tilde{\mathbf{q}}=\left(\mathbf{n} \sin \frac{\phi}{2}, \cos \frac{\phi}{2}\right)^{T}=\left(\tilde{q}_{0}, \tilde{q}_{1}, \tilde{q}_{2}, \tilde{q}_{3}\right)^{T}
$$

where $\mathbf{n}$ is the rotation axis and $\phi$ is the rotation angle about the roation axis. For common use, one should convert it to regular form. Hence the final unitary quaternion solution is given by

$$
\mathbf{q}=\left(\cos \frac{\phi}{2}, \mathbf{n} \sin \frac{\phi}{2}\right)^{T}=\frac{\left(\tilde{q}_{3}, \tilde{q}_{0}, \tilde{q}_{1}, \tilde{q}_{2}\right)^{T}}{\sqrt{\tilde{q}_{0}^{2}+\tilde{q}_{1}^{2}+\tilde{q}_{2}^{2}+\tilde{q}_{3}^{2}}} .
$$

\section{EQUiVALENCE ANALYSIS}

Equation (16) is in fact a extremely simplified solution to the Wahba's problem. Therefore, combining Markley's two-vector approach or other Wahba's solutions with (12) gives the same numerical solution of attitude quaternion shown in (16).
The overwhelming factor to obtain the proposed simple solution presented in (16) is indeed (12). In this section we are going to show that for representative existing magnetometer reference vector free algorithms, this preliminary always holds. Let us review the computation procedures of FQA and AQUA [9], [11]. The FQA extracts the roll and pitch via the accelerometer measurements using elementary quaternions:

$$
\begin{aligned}
& \text { pitch }:\left\{\begin{array}{l}
a_{x}=\sin \theta \\
\mathbf{q}_{\text {pitch }}=\left(\cos \frac{\theta}{2}, 0, \sin \frac{\theta}{2}, 0\right)^{T}
\end{array}\right. \\
& \text { roll }:\left\{\begin{array}{l}
\sin \varphi=-\frac{a_{y}}{\cos \theta} \\
\cos \varphi=-\frac{a_{z}}{\cos \theta} \\
\mathbf{q}_{\text {roll }}=\left(\cos \frac{\varphi}{2}, \sin \frac{\varphi}{2}, 0,0\right)^{T}
\end{array}\right.
\end{aligned}
$$

where $\theta, \varphi$ denote the pitch and roll angles respectively. $\mathbf{q}_{\text {roll }}$ and $\mathbf{q}_{\text {pitch }}$ are independently estimated quaternions for two rotation directions roll and pitch. AQUA solves the joint quaternion of roll and pitch by an indefinite system of equations using acceleration attitude kinematics:

$$
\left\{\begin{array}{c}
2\left(q_{1} q_{3}+q_{0} q_{2}\right)=a_{x} \\
2\left(q_{2} q_{3}-q_{0} q_{1}\right)=a_{y} \\
q_{0}^{1}-q_{1}^{2}-q_{2}^{2}+q_{3}^{2}=a_{z}
\end{array}\right.
$$

This leads to the general solution of

$$
\mathbf{q}_{a c c}= \begin{cases}\left(\kappa_{1},-\frac{a_{y}}{2 \kappa_{1}}, \frac{a_{x}}{2 \kappa_{1}}, 0\right)^{T}, & a_{z} \geqslant 0 \\ \left(-\frac{a_{y}}{2 \kappa_{2}}, \kappa_{2}, 0, \frac{a_{x}}{2 \kappa_{2}}\right)^{T}, & a_{z}<0\end{cases}
$$

where

$$
\begin{aligned}
& \kappa_{1}=\sqrt{\frac{a_{z}+1}{2}} \\
& \kappa_{2}=\sqrt{\frac{1-a_{z}}{2}}
\end{aligned}
$$

Therefore the first stages of estimating the non-yaw angles belonging to FQA and AQUA respectively are very similar and they have the same accuracy in principle. Then the two algorithms all rotate the magnetometer measurements into an intermediate earth frame avoiding using local magnetic reference vector. Differently, they are performed by means of quaternion multiplication

$$
\left(\begin{array}{c}
0 \\
\tilde{\mathbf{M}}
\end{array}\right)=\mathbf{q}_{\text {pitch }} \otimes \mathbf{q}_{\text {roll }} \otimes\left(\begin{array}{c}
0 \\
\mathbf{M}^{b}
\end{array}\right) \otimes \mathbf{q}_{\text {roll }}^{-1} \otimes \mathbf{q}_{\text {pitch }}^{-1}
$$

for FQA. And DCM rotation by AQUA, such that

$$
\mathbf{C}^{T}\left(\mathbf{q}_{a c c}\right) \mathbf{M}^{b}=\tilde{\mathbf{M}}
$$

where $\otimes$ denotes the quaternion product; $\tilde{\mathbf{M}}=\left(h_{x}, h_{y}, h_{z}\right)^{T}$ is the temporary magnetometer reference vector lying on the intermediate frame. The yaw quaternion is then estimated by satisfying

$$
\left(0, \sqrt{h_{x}^{2}+h_{y}^{2}}, 0, h_{z}\right)^{T}=\mathbf{q}_{\text {yaw }} \otimes\left(\begin{array}{c}
0 \\
\tilde{\mathbf{M}}
\end{array}\right) \otimes \mathbf{q}_{\text {yaw }}^{-1}
$$

or

$$
\mathbf{C}^{T}\left(\mathbf{q}_{\text {mag }}\right) \tilde{\mathbf{M}}=\left(\sqrt{h_{x}^{2}+h_{y}^{2}}, 0, h_{z}\right)^{T}
$$


TABLE I

FLOPS OF ALGORITHMS

\begin{tabular}{cccccc}
\hline Algorithm & + & $\times$ & $/$ & $\sqrt{\cdots}$ & $\cos (\cdots)$ \\
\hline Proposed SAAM & $\mathbf{1 8}$ & $\mathbf{1 6}$ & $\mathbf{1}$ & $\mathbf{2}$ & $\mathbf{0}$ \\
FQA & 18 & 53 & 4 & 3 & 6 \\
Markley & 33 & 51 & 3 & 4 & 0 \\
AQUA & 24 & 38 & 2 & 4 & 0 \\
\hline
\end{tabular}

The final full-attitude quaternion is formed by quaternion multiplication and singularity avoidance. This shows that FQA and AQUA are the same in principle. Note that in fact

$$
\left(\sqrt{h_{x}^{2}+h_{y}^{2}}, 0, h_{z}\right)^{T}=\mathbf{M}^{r}
$$

A key step of the above algorithms is to compute $\tilde{\mathbf{M}}$. From (26), one can find out that

$$
\mathbf{C}^{T}\left(\mathbf{q}_{\text {mag }}\right) \mathbf{C}^{T}\left(\mathbf{q}_{a c c}\right) \mathbf{M}^{b}=\left(\sqrt{h_{x}^{2}+h_{y}^{2}}, 0, h_{z}\right)^{T}
$$

Note that

$$
\mathbf{C}^{T}\left(\mathbf{q}_{\text {mag }}\right) \mathbf{C}^{T}\left(\mathbf{q}_{a c c}\right)=\mathbf{C}^{T}(\mathbf{q})
$$

is actually the full-attitude rotation allowing for $\mathbf{M}^{b}=$ $\mathbf{C}(\mathbf{q}) \mathbf{M}^{r}$. This shows that the FQA and AQUA are essentially the same with Wahba's optimization for accelerometer and magnetometer. Then it is ensured that (12) holds for these methods as well.

Yun's FQA and Valenti's AQUA are both algebraic methods free of magnetometer reference vectors. In general, AQUA and FQA extracts the maximum information from accelerometer and magnetometer measurements, which has been systematically verified in last sub-section. From the presented approach shown above, we can see that the proposed method is consistent with AQUA and FQA on this point owning the internal dynamics of (12). Hence, the proposed algorithm, Markley's two-vector method, AQUA and FQA have the same attitude determination accuracy. As they employ quite different architectures, the computation speeds vary a lot. The computation complexity analysis and execution time evaluation will be presented in the following sections.

\section{Computation Complexity}

The proposed method owns the simplest form of quaternion as we know. Then the algorithm is named as the Super-fast Attitude of Accelerometer and Magnetometer (SAAM). In this section, we are going to give statistics of floating-number operations (FLOPS) and exact attitude tests. Representatives like FQA, Markley's two-vector method and AQUA are introduced for comparisons. The FLOPS of various algorithms are presented in Table I.

For embedded platforms without floating-point number unit (FPU, [29]), floating number (IEEE-754 standard, [30]) operations are processed by the CPU. In such occasion, addition and multiplication are done instantaneously in one machine processing cycle. One floating-number square-root operation requires approximately 7 cycles while that of sine or cosine function are 15 cycles [31]. The division is usually $33 \%$
TABLE II

Time Complexities of Algorithms

\begin{tabular}{ccc}
\hline Algorithm & Without FPU & With FPU \\
\hline Proposed SAAM & $\mathbf{O}(\mathbf{4 9 . 5 n})$ & $\mathbf{O}(\mathbf{3 5 . 5 n})$ \\
FQA & $O(188.0 n)$ & $O(83.0 n)$ \\
Markley & $O(116.5 n)$ & $O(85.5 n)$ \\
AQUA & $O(93.0 n$ & $O(63.5 n)$ \\
\hline
\end{tabular}

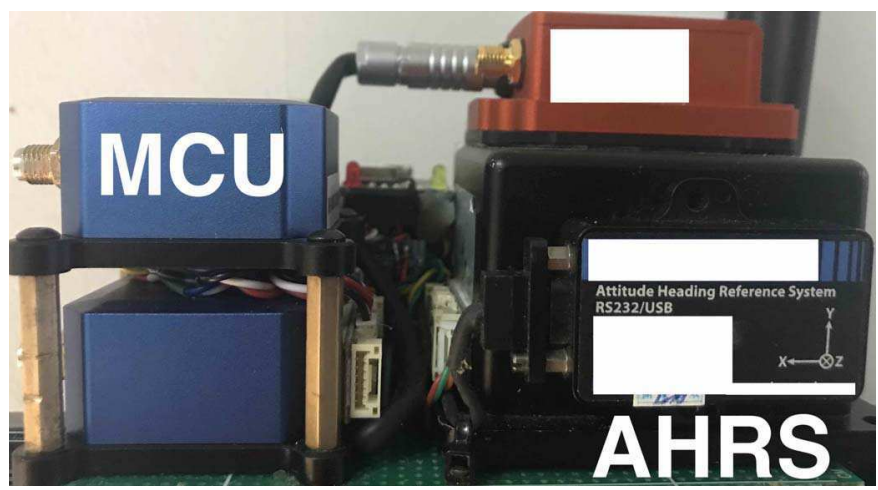

Fig. 1. Designed hardware for validation.

slower than addition or multiplication. When FPU takes place, the performance of square root and sine, cosine could be optimized using DSP for saving at most 95\% computation time [32]. Now, let us define we have $n$ loops to conduct. Then the time complexity of the algorithms with or without FPU can be calculated in Table II. Therefore, via the comparisons shown above, the theoretical algorithm advance in the speed is about $47.3 \%$ to $73.7 \%$ without FPU and $47.2 \%$ to $58.5 \%$ with FPU.

\section{HARDWARE VALIDATION}

The algorithms are executed on a commercial attitude and heading reference system (AHRS). The AHRS is connected to our 32-bit micro controller unit (MCU) with the processing unit of $400 \mathrm{MHz}$ core clock speed with FPU, via the serial port under the baudrate of $921600 \mathrm{bps}$ and sampling frequency of $500 \mathrm{~Hz}$. The internal digital low pass filter (DLPF) is tuned to be with cut-off frequency of $66.6 \mathrm{~Hz}$ for default setting. All the numerical modules, e.g., square root and trigonometric functions are implemented using the public library offered by the hardware vendor. The hardware setup is shown in Fig. 1.

A record of raw sensor data and reference angles are logged with the MCU and the calculation is conducted on the chip with an embedded operating system called the FreeRTOS at the kernel scheduling frequency of $1000 \mathrm{~Hz}$. The scheduling method of the RTOS is set to the Round-Robin one that makes every task run synchronously [33]. Each algorithm is written into a task of scheduling frequency of $200 \mathrm{~Hz}$ with the same priority. The computed attitude angles' root meansquared errors (RMSEs) along with the statistics are shown in Fig. 2 and Table III. It is noted that the RMSE is computed with the reference Euler angles output from the AHRS.

Also, with the aid of the internal high resolution timer, the execution time consumption on chip is collected. The results 

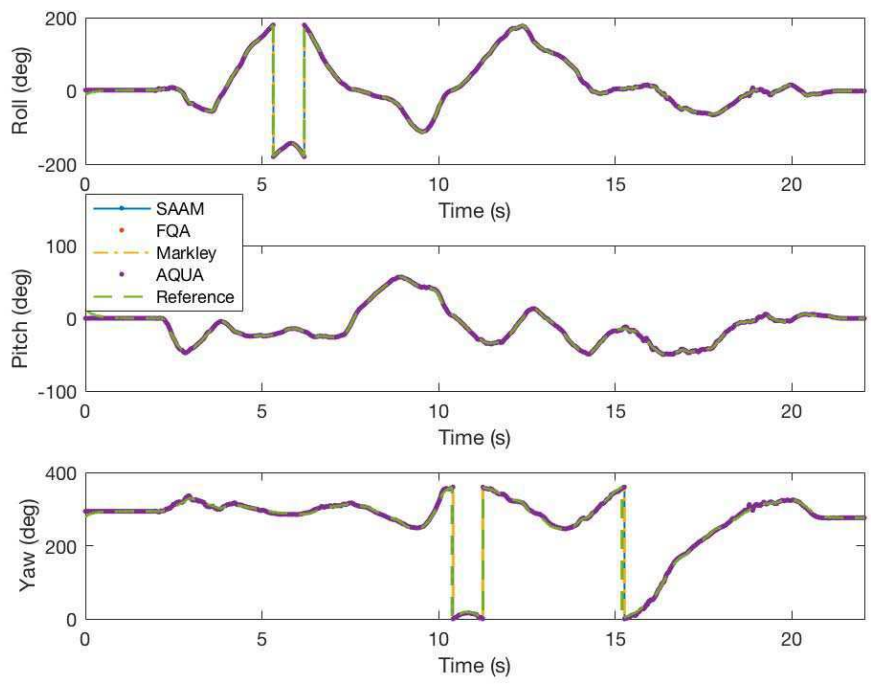

Fig. 2. The full-attitude test result.

TABLE III

RMSES OF ATTITUDE ANGLES

\begin{tabular}{cccc}
\hline Algorithm & Roll $(\mathrm{deg})$ & Pitch $(\mathrm{deg})$ & Yaw $(\mathrm{deg})$ \\
\hline Proposed SAAM & $\mathbf{7 . 0 0 8 4 4 1 9 5 4 2 1 1 0 3 2}$ & $\mathbf{1 . 1 9 0 5 2 9 2 2 8 8 5 3 9 0 2}$ & $\mathbf{2 4 . 7 3 2 7 8 1 3 1 6 9 3 5 1 4 2}$ \\
FQA & 7.008441954211161 & 1.1905292288593916 & 24.732781316335156 \\
Markley & 7.008441954211033 & 1.190529228853914 & 24.732781316935142 \\
AQUA & 7.008441954211031 & 1.190529228853916 & 24.732781316935155 \\
\hline
\end{tabular}

TABLE IV

EXECUTION TIME CONSUMPTION RESUlt

\begin{tabular}{ccc}
\hline Algorithm & Time $(\mathrm{s})$ & Standard Deviation $(\mathrm{s})$ \\
\hline Proposed SAAM & $\mathbf{1 . 7 7 2 3 9} \times \mathbf{1 0}^{-\mathbf{6}}$ & $\mathbf{4 . 7 0 8 7 7} \times \mathbf{1 0}^{-\mathbf{7}}$ \\
FQA & $1.18861 \times 10^{-5}$ & $1.40646 \times 10^{-6}$ \\
Markley & $9.10740 \times 10^{-6}$ & $1.01356 \times 10^{-6}$ \\
AQUA & $6.58190 \times 10^{-6}$ & $8.17700 \times 10^{-7}$ \\
\hline
\end{tabular}

are summarized in Table IV where the standard deviation indicates the time consistency of a certain algorithm. From the results we can see that the proposed SAAM maintains the attitude determination accuracy and significantly reduces the computation time. The general speed of the SAAM is about $400 \sim 660 \%$ times that of existing algorithms. The test results on embedded computer basically coincide with the theoretical time complexity analysis in Section IV which verifies the correctness as well.

\section{DISCUSSION}

The proposed SAAM is the fastest one compared with stateof-the-art algorithms. The saved CPU time can be used for less power, more effective scheduling, smarter failsafe of system and etc. As a matter of fact, from (16), we can see that it is the simple form that consumes the least execution time. This simple computation procedure also helps hardware engineers easily implement the circuit of this algorithm. Here, for example, when designing by means of digital circuit tools, some computations, e.g., square root and cosine functions are not easy to be implemented [34]. In the final stage of the algorithmic validation, we have designed the real-world integrated circuit (IC). The current state of the circuit implementation indicates that much less electronic components are required with the proposed SAAM algorithm. The designed scheme has allowed for longer lifetime of devices in use without losing accuracy, according to its extreme low power consumption. Therefore, with the current obtained results, we hope that we may design a simple and cheap digital solution for combined attitude determination from gyroscope, accelerometer and magnetometer for more general requirements and mass production of IC in the future.

\section{CONCLUSION}

In this paper, a novel solution to the attitude determination from accelerometer and magnetometer is proposed. We name it the SAAM algorithm, which employs the simplest quaternion form ever seen. Mandatory derivations are shown to derive the analytic solution. It is performed that the proposed SAAM is equivalent to FQA, AQUA and Markley's algorithm. The superiority of the algorithm is analysed via numerical evaluation of FLOPS and time complexity. The final experiment shows that the proposed SAAM has almost the same accuracy but consumes much less execution time, compared with representative algorithms. We hope that SAAM will boost the embedded attitude system design of accelerometer and magnetometer in the future.

\section{APPENDIX A \\ ANALYTICAL DERIVATIONS}

Coefficients of Characteristic Polynomial: Using (7) and (1), we can compute the coefficients by

$$
\begin{aligned}
\tau_{1}= & 4(w-1) w a_{x} m_{D} m_{x}+4(w-1) w a_{y} m_{D} m_{y} \\
& +4(w-1) w a_{z} m_{D} m_{z}-4 w^{2}+4 w-2 \\
\tau_{2}= & 0 \\
\tau_{3}= & 1-8(w-1) w^{3} a_{x} m_{D} m_{x}-8(w-1) w^{3} a_{y} m_{D} m_{y} \\
& -8(w-1) w^{3} a_{z} m_{D} m_{z}+8(w-1) w^{2} a_{x} m_{D} m_{x} \\
& +8(w-1) w^{2} a_{y} m_{D} m_{y}+8(w-1) w^{2} a_{z} m_{D} m_{z} \\
& -4(w-1) w a_{x} m_{D} m_{x}-4(w-1) w a_{y} m_{D} m_{y} \\
& -4(w-1) w a_{z} m_{D} m_{z}+8(w-1)^{2} w^{2} a_{x} a_{y} m_{x} m_{y} \\
& +8(w-1)^{2} w^{2} a_{x} a_{z} m_{x} m_{z}+4(w-1)^{2} w^{2} a_{z}^{2} m_{y}^{2} \\
& +4(w-1)^{2} w^{2} a_{y}^{2} m_{z}^{2}+8(w-1)^{2} w^{2} a_{y} a_{z} m_{y} m_{z} \\
& +8(w-1)^{2} w^{2} a_{y}^{2} m_{y}^{2}+8(w-1)^{2} w^{2} a_{z}^{2} m_{z}^{2} \\
& -4(w-1)^{2} w^{2} a_{y}^{2}-4(w-1)^{2} w^{2} a_{z}^{2} \\
& -4(w-1)^{2} w^{2} m_{N}^{2}-4(w-1)^{2} w^{2} m_{y}^{2} \\
& -(w-1)^{2} w^{2} m_{z}^{2}+8 w^{4}-16 w^{3}+12 w^{2}-4 w
\end{aligned}
$$

Repeating invoking (1) and (10) generates the results in (8).

\section{APPENDIX B \\ EIGENVALUES}

The eigenvalues of $\mathbf{K}$ can be solved via the roots of its characteristic polynomial. Such eigenvalues are give by

$$
\lambda_{\mathbf{K}, 1,2,3,4}= \pm \sqrt{\xi_{1} \pm \xi_{2}}
$$


where

$$
\begin{aligned}
\xi_{1}= & 2 \sqrt{m_{N}{ }^{2}\left(w^{2}-w\right)^{2}\left(\begin{array}{l}
a_{x}{ }^{2} m_{y}{ }^{2}+a_{x}{ }^{2} m_{z}{ }^{2}-2 a_{x} a_{y} m_{x} m_{y} \\
-2 a_{x} a_{z} m_{x} m_{z}+a_{y}{ }^{2} m_{x}{ }^{2}+a_{y}{ }^{2} m_{z}{ }^{2} \\
-2 a_{y} a_{z} m_{y} m_{z}+a_{z}{ }^{2} m_{x}{ }^{2}+a_{z}{ }^{2} m_{y}{ }^{2}
\end{array}\right)} \\
\xi_{2}= & a_{x}{ }^{2} w^{2}-2 a_{x} m_{D} m_{x} w^{2}+2 a_{x} m_{D} m_{x} w+a_{y}{ }^{2} w^{2} \\
& -2 a_{y} m_{D} m_{y} w^{2}+2 a_{y} m_{D} m_{y} w+a_{z}{ }^{2} w^{2}-2 a_{z} m_{D} m_{z} w^{2} \\
& +2 a_{z} m_{D} m_{z} w+m_{D}{ }^{2} m_{x}{ }^{2} w^{2}-2 m_{D}{ }^{2} m_{x}{ }^{2} w+m_{D}{ }^{2} m_{x}{ }^{2} \\
& +m_{D}{ }^{2} m_{y}{ }^{2} w^{2}-2 m_{D}{ }^{2} m_{y}{ }^{2} w+m_{D}{ }^{2} m_{y}{ }^{2}+m_{D}{ }^{2} m_{z}{ }^{2} w^{2} \\
& -2 m_{D}{ }^{2} m_{z}{ }^{2} w+m_{D}{ }^{2} m_{z}{ }^{2}+m_{N}{ }^{2} m_{x}{ }^{2} w^{2}-2 m_{N}{ }^{2} m_{x}{ }^{2} w \\
& +m_{N}{ }^{2} m_{x}{ }^{2}+m_{N}{ }^{2} m_{y}{ }^{2} w^{2}-2 m_{N}{ }^{2} m_{y}{ }^{2} w+m_{N}{ }^{2} m_{y}{ }^{2} \\
& +m_{N}{ }^{2} m_{z}{ }^{2} w^{2}-2 m_{N}{ }^{2} m_{z}{ }^{2} w+m_{N}{ }^{2} m_{z}{ }^{2}
\end{aligned}
$$

Inserting (1) into it, we have

$$
\begin{aligned}
\xi_{1}=2 \sqrt{m_{N}^{2}(w-1)^{2} w^{2}\left[\begin{array}{l}
a_{x}{ }^{2}\left(m_{y}{ }^{2}+m_{z}^{2}\right) \\
-2 a_{y} m_{y}\left(a_{x} m_{x}+a_{z} m_{z}\right) \\
-2 a_{x} a_{z} m_{x} m_{z} \\
+a_{y}{ }^{2}\left(m_{x}^{2}+m_{z}^{2}\right) \\
+a_{z}^{2}\left(m_{x}^{2}+m_{y}^{2}\right)
\end{array}\right]} \\
\xi_{2}=1-2 w-2 a_{x} m_{D} m_{x} w(w-1)-2 a_{y} m_{D} m_{y} w(w-1)
\end{aligned}
$$

Combining

$$
\begin{aligned}
\alpha^{2}= & \left(a_{x} m_{x}+a_{y} m_{y}+a_{z} m_{z}\right)^{2} \\
= & a_{x}^{2} m_{x}^{2}+a_{y}^{2} m_{y}^{2}+a_{z}^{2} m_{z}^{2}+2 a_{x} a_{y} m_{x} m_{y} \\
& +2 a_{x} a_{z} m_{x} m_{z}+2 a_{y} a_{z} m_{y} m_{z} \\
= & a_{x}^{2}\left(1-m_{y}^{2}-m_{z}^{2}\right)+a_{y}^{2}\left(1-m_{x}^{2}-m_{z}^{2}\right) \\
& +a_{z}^{2}\left(1-m_{x}^{2}-m_{y}^{2}\right)+2 a_{x} a_{y} m_{x} m_{y} \\
& +2 a_{x} a_{z} m_{x} m_{z}+2 a_{y} a_{z} m_{y} m_{z} \\
= & 1-a_{x}^{2}\left(m_{y}^{2}+m_{z}^{2}\right)-a_{y}^{2}\left(m_{x}^{2}+m_{z}^{2}\right) \\
& -a_{z}^{2}\left(m_{x}^{2}+m_{y}^{2}\right)+2 a_{x} a_{y} m_{x} m_{y} \\
& +2 a_{x} a_{z} m_{x} m_{z}+2 a_{y} a_{z} m_{y} m_{z}
\end{aligned}
$$

with the above equation (33) gives (9).

\section{ACKNOWLEDGMENT}

The source code of the paper has been uploaded to https://github.com/zarathustr/SAAM for public evaluation.

\section{REFERENCES}

[1] E. Choi and S. Chang, "A consumer tracking estimator for vehicles in GPS-free environments," IEEE Trans. Consum. Electron., vol. 63, no. 4, pp. 450-458, Nov. 2017.

[2] X. Yun and E. R. Bachmann, "Design, implementation, and experimental results of a quaternion-based Kalman filter for human body motion tracking," IEEE Trans. Robot., vol. 22, no. 6, pp. 1216-1227, Dec. 2006

[3] J. Gośliński, M. Nowicki, and P. Skrzypczyński, "Performance comparison of EKF-based algorithms for orientation estimation on android platform," IEEE Sensors J., vol. 15, no. 7, pp. 3781-3792, Jul. 2015.

[4] Z. Zhou, Y. Li, J. Zhang, and C. Rizos, "Integrated navigation system for a low-cost quadrotor aerial vehicle in the presence of rotor influences," J. Surv. Eng., vol. 143, no. 1, 2017, Art. no. 05016006.

[5] S.-D. Choi and S.-Y. Lee, "3D stroke reconstruction and cursive script recognition with magnetometer-aided inertial measurement unit," IEEE Trans. Consum. Electron., vol. 58, no. 2, pp. 661-669, May 2012.
[6] W. Li and J. Wang, "Effective adaptive Kalman filter for MEMSIMU/magnetometers integrated attitude and heading reference systems," J. Navig., vol. 66, no. 1, pp. 99-113, 2012.

[7] W. Li, J. Wang, and Y. Wo, "Magnetic sensors for navigation applications: An overview," J. Navig., vol. 67, no. 2, pp. 263-275, 2014.

[8] J. Wu et al., "Fast linear quaternion attitude estimator using vector observations," IEEE Trans. Autom. Sci. Eng., vol. 15, no. 1, pp. 307-319, Jan. 2018.

[9] X. Yun, E. R. Bachmann, and R. B. McGhee, "A simplified quaternionbased algorithm for orientation estimation from earth gravity and magnetic field measurements," IEEE Trans. Instrum. Meas., vol. 57 no. 3, pp. 638-650, Mar. 2008.

[10] F. L. Markley, "Fast quaternion attitude estimation from two vector measurements," J. Guid. Control Dyn., vol. 25, no. 2, pp. 411-414, 2002.

[11] R. G. Valenti, I. Dryanovski, and J. Xiao, "A linear Kalman filter for MARG orientation estimation using the algebraic quaternion algorithm,' IEEE Trans. Instrum. Meas., vol. 65, no. 2, pp. 467-481, Feb. 2016.

[12] J. Wu, T. Wang, Z. Zhou, H. Yin, and R. Li, "Analytic accelerometermagnetometer attitude determination without reference information," Int. J. Micro Air Veh., Feb. 2018, doi: 10.1177/1756829318756356.

[13] P. Marantos, Y. Koveos, and K. J. Kyriakopoulos, "UAV state estimation using adaptive complementary filters," IEEE Trans. Control Syst. Technol., vol. 24, no. 4, pp. 1214-1226, Jul. 2016.

[14] H. Fourati, N. Manamanni, L. Afilal, and Y. Handrich, "Complementary observer for body segments motion capturing by inertial and magnetic sensors," IEEE/ASME Trans. Mechatronics, vol. 19, no. 1, pp. 149-157, Feb. 2014.

[15] L. Chang, B. Hu, and G. Chang, "Modified unscented quaternion estimator based on quaternion averaging," J. Guid. Control Dyn., vol. 37, no. 1, pp. 305-308, 2014.

[16] Y. Huang, Y. Zhang, and X. Wang, "Kalman-filtering-based in-motion coarse alignment for odometer-aided SINS," IEEE Trans. Instrum. Meas., vol. 66, no. 12, pp. 3364-3377, Dec. 2017.

[17] Z. Zhou, Y. Li, J. Liu, and G. Li, "Equality constrained robust measurement fusion for adaptive Kalman-filter-based heterogeneous multisensor navigation," IEEE Trans. Aerosp. Electron. Syst., vol. 49, no. 4, pp. 2146-2157, Oct. 2013.

[18] B. Lee, W.-C. Bang, J. D. K. Kim, and C. Y. Kim, "Orientation estimation in mobile virtual environments with inertial sensors," IEEE Trans. Consum. Electron., vol. 57, no. 2, pp. 802-810, May 2011.

[19] A. Makni, H. Fourati, and A. Y. Kibangou, "Energy-aware adaptive attitude estimation under external acceleration for pedestrian navigation," IEEE/ASME Trans. Mechatronics, vol. 21, no. 3, pp. 1366-1375, Jun. 2016.

[20] J. Wu, Z. Zhou, R. Li, L. Yang, and H. Fourati, "Attitude determination using a single sensor observation: Analytic quaternion solutions and property discussion," IET Sci. Meas. Technol., vol. 11, no. 3, pp. 731-739, Sep. 2017.

[21] S. O. H. Madgwick, A. J. L. Harrison, and R. Vaidyanathan, "Estimation of IMU and MARG orientation using a gradient descent algorithm," in Proc. IEEE Int. Conf. Rehabil. Robot., 2011, pp. 1-7.

[22] P. Zagorski, T. Dziwinski, and A. Tutaj, "Steepest descent quaternion attitude estimator," Aerosp. Sci. Technol., vol. 77, pp. 1-10, Jun. 2018.

[23] Y. Tian, H. Wei, and J. Tan, "An adaptive-gain complementary filter for real-time human motion tracking with MARG sensors in free-living environments," IEEE Trans. Neural Syst. Rehabil. Eng., vol. 21, no. 2, pp. 254-264, Mar. 2013.

[24] H. Fourati, N. Manamanni, L. Afilal, and Y. Handrich, "A nonlinear filtering approach for the attitude and dynamic body acceleration estimation based on inertial and magnetic sensors: Bio-logging application," IEEE Sensors J., vol. 11, no. 1, pp. 233-244, Jan. 2011.

[25] F. L. Markley and J. L. Crassidis, Fundamentals of Spacecraft Attitude Determination and Control, vol. 2. New York, NY, USA: Springer, 2014.

[26] Y. Yang and Z. Zhou, "An analytic solution to Wahba's problem," Aerosp. Sci. Technol., vol. 30, no. 1, pp. 46-49, 2013.

[27] J. Wu, Z. Zhou, J. Chen, H. Fourati, and R. Li, "Fast complementary filter for attitude estimation using low-cost MARG sensors," IEEE Sensors J., vol. 16, no. 18, pp. 6997-7007, Sep. 2016.

[28] M. D. Shuster and S. D. Oh, "Three-axis attitude determination from vector observations," J. Guid. Control Dyn., vol. 4, no. 1, pp. 70-77, 1981.

[29] J. Yiu, The Definitive Guide to ARM Cortex-M3 and Cortex-M4 Processors. Amsterdam, The Netherlands: Newnes, 2013.

[30] M. G. Arnold, T. A. Bailey, J. R. Cowles, and M. D. Winkel, "Applying features of IEEE 754 to sign/logarithm arithmetic," IEEE Trans. Comput., vol. 41, no. 8, pp. 1040-1050, Aug. 1992. 
[31] A. Van Someren and C. Atack, The ARM RISC Chip: A Programmer's Guide. Wokingham, U.K.: Addison-Wesley, 1994.

[32] R. M. Stallman, Using and Porting the GNU Compiler Collection. Boston, MA, USA: Free Softw. Found., 2009.

[33] E. L. Hahne, "Round-Robin scheduling for max-min fairness in data networks," IEEE J. Sel. Areas Commun., vol. 9, no. 7, pp. 1024-1039, Sep. 1991.

[34] H. Fu, W. Osborne, R. Clapp, O. Mencer, and W. Luk, "Accelerating seismic computations using customized number representations on FPGAs," EURASIP J. Embedded Syst., vol. 2009, no. 1, 2008, Art. no. 382983.

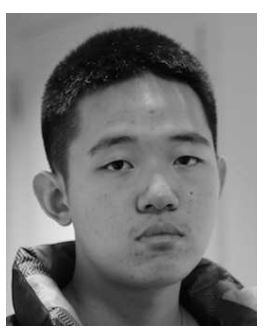

Jin Wu (M'17) received the B.S. degree in automation engineering from the University of Electronic Science and Technology of China, Chengdu, China, where he is currently a Research Assistant with the School of Aeronautics and Astronautics.

His research interests include inertial navigation, optimal filtering, control theory, and robot vision. He has published over 10 technical papers on many academic journals, such as IEEE TRANSACTIONS, IEEE Journals, and IET Journals.

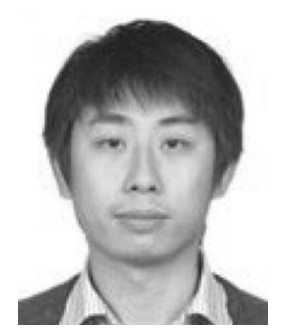

Zebo Zhou received the B.Sc. and M.Sc. degrees in surveying and engineering from Wuhan University in 2004 and 2006, respectively, and the Ph.D. degree with the College of Surveying and GeoInformatics, Tongji University, Shanghai, China, in 2009. He is currently an Associate Professor with the School of Aeronautics and Astronautics, University of Electronic Science and Technology of China. His research interests include GNSS navigation and positioning, GNSS/INS integration, multisensor fusion, and surveying data processing theory.

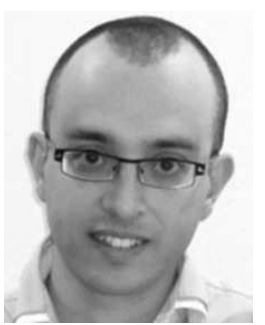

Hassen Fourati received the Bachelor of Engineering degree in electrical engineering from the National Engineering School of Sfax, Tunisia, the master's degree in automated systems and control from the University Claude Bernard, Lyon, France, and the Ph.D. degree in automatic control form the University of Strasbourg, France, in 2006, 2007, and 2010, respectively. He is currently an Associate Professor with the Electrical Engineering and Computer Science, University Grenoble Alpes, Grenoble, France, and a member of the Networked Controlled Systems Team, affiliated to the Automatic Control Department of the GIPSA-Lab. His research interests include nonlinear filtering and estimation and multisensor fusion with applications in navigation, robotics, and traffic management.

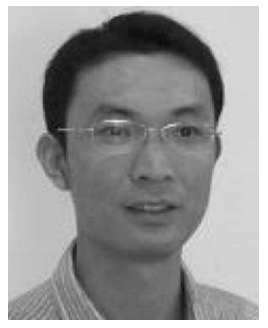

Yuhua Cheng (M'11-SM'13) received the M.S. and $\mathrm{Ph} . \mathrm{D}$. degrees in measurement techniques and automation devices from Sichuan University, Chengdu, China.

$\mathrm{He}$ is currently with the University of Electronic Science and Technology of China, Chengdu, where he is a Professor with the School of Automation. His research relates to nondestructive evaluation, structural health monitoring, the application of photonics in sensing and communications systems and wireless sensor networks. He has been serving as an Associate Editor for the IEEE TRANSACTIONS ON INSTRUMENTATION AND MEASUREMENT since 2017. 